\title{
Genetic diversity of chloroquine-resistant Plasmodium vivax parasites from the western Brazilian Amazon
}

\author{
Omaira Vera Lizcano ${ }^{1 /+}$, Sarah Stela Resende ${ }^{2}$, Yonne F Chehuan ${ }^{3}$, \\ Marcus VG Lacerda ${ }^{3}$, Cristiana FA Brito², Mariano G Zalis ${ }^{1}$
}

\begin{abstract}
'Laboratório de Infectologia e Parasitologia Molecular, Serviço de Patologia Clínica, Hospital Universitário Clementino Fraga Filho, Universidade Federal do Rio de Janeiro, Rio de Janeiro, RJ, Brasil '2Laboratório de Malária, Centro de Pesquisa René Rachou-Fiocruz, Belo Horizonte, MG, Brasil ${ }^{3}$ Fundação de Medicina Tropical Dr Heitor Vieira Dourado, Manaus, AM, Brasil
\end{abstract}

The molecular basis of Plasmodium vivax chloroquine (CQ) resistance is still unknown. Elucidating the molecular background of parasites that are sensitive or resistant to CQ will help to identify and monitor the spread of resistance. By genotyping a panel of molecular markers, we demonstrate a similar genetic variability between in vitro CQ-resistant and sensitive phenotypes of $\mathrm{P}$. vivax parasites. However, our studies identified two loci (MS8 and MSP1-B10) that could be used to discriminate between both CQ-susceptible phenotypes among P. vivax isolates in vitro. These preliminary data suggest that microsatellites may be used to identify and to monitor the spread of $\mathrm{P}$. vivax-resistance around the world.

Key words: Plasmodium vivax - chloroquine - resistance

Plasmodium vivax, although less pathogenic than Plasmodium falciparum, has a great socioeconomic impact. $P$. vivax has been associated with drug resistance, clinical severity and even fatality (Price et al. 2009). The first cases of $P$. vivax chloroquine resistance (CQ-R) were reported in 1989 in Papua New Guinea (Rieckmann et al. 1989). Since then, reports of CQ-R have been published around the world. Some cases of CQ-R that presented adequate blood CQ levels had been confirmed in Brazil, Ethiopia, Indonesia, Malaysia, Myanmar, Papua New Guinea, Peru, the Solomon Islands and Thailand (WHO 2010). In Brazil, malaria is transmitted along the Amazon Basin. A total of 242,758 cases were reported in 2012, with $P$. vivax accounting for $85 \%$ of all cases (WHO 2013). The first reliable case of in vivo CQ-R in Brazil was reported in Manaus, state of Amazonas (AM) (Alecrim et al. 1999). In 2004/2005, 10\% of $P$. vivax infections studied in Manaus showed an in vivo CQ-resistant (CQ-Rt) phenotype (Santana Filho et al. 2007). Recently, two studies evaluated $P$. vivax CQ-susceptibility by using short-term in vitro cultures of samples collected in 2004-2008 from AM and confirmed the same prevalence of CQ-R among $P$. vivax parasites $(10 \%)$ (Chehuan et al. 2013, Pratt-Riccio et al. 2013). In other endemic regions of Brazil, such as in the state of Acre, no CQ-R has been reported (Orjuela-Sánchez et al. 2009) and no studies have been reported so far in other regions of Latin America (Gonçalves et al. 2014).

\footnotetext{
doi: 10.1590/0074-0276140241

Financial support: CAPES, FAPEAM, CNPq

$\mathrm{CFAB}$ and MVGL receive scholarship from CNPq.

+ Corresponding author: vera.omaira@gmail.com

Received 9 July 2014

Accepted 1 October 2014
}

The genetic mechanism of $P$. vivax CQ-R has yet to be fully elucidated. While CQ-R in P. falciparum has been associated with particular point mutations in specific genes, such as pfcrt-o and pfmdr-1 (Fidock et al. 2000 , Duraisingh \& Cowman 2005), this type of association is not very clear in the case of $P$. vivax (Gonçalves et al. 2014). The main difficulty in studying the mechanisms of $P$. vivax CQ-R is the absence of a continuous in vitro culture system, as studies utilising short-term in vitro cultures are useful for investigating drug resistance mechanisms and susceptibility analysis of therapeutic agents for $P$. vivax (Kerlin et al. 2012). Due to the absence of specific molecular markers of resistance, the characterisation of general molecular markers of $P$. vivax will not only help identify CQ-R parasites, but also define the geographical origins and dissemination of resistant isolates (Arnott et al. 2012). The genetic diversity of $P$. vivax has been studied using nearly neutral molecular markers, such as microsatellites (MS) and tandem repeats (TR), as well as with polymorphic antigens, including the PvMSP protein family members (MSP-1 and MSP-3 $\alpha$ ) [for a review see de Brito and Ferreira (2011)]. The present study focuses on the genetic diversity of $P$. vivax isolates from the Brazilian Amazon Basin with different CQ-susceptibility phenotypes in short-term in vitro cultures.

Patients with uncomplicated $P$. vivax malaria, as confirmed by a thick blood smear, were randomly selected in the outpatient clinics Dr Heitor Vieira Dourado Tropical Medicine Foundation (Manaus) between December 2007-July 2008. Written informed consent was obtained from all participating patients. The major exclusion criterion was the use of antimalarials within the previous 60 days. Blood was collected at the day of diagnosis and before the start of antimalarial treatment. The patients were treated according to the Brazilian Ministry of Health standards for malaria therapy (Chehuan et al. 2013). Eleven isolates from the Amazon Basin were evaluated: nine from AM, one from the state 
TABLE I

Molecular markers characterisation

\begin{tabular}{lcccc}
\hline Marker & $\begin{array}{c}\text { First } \\
\text { position }\end{array}$ & Chromosome & $\begin{array}{c}\text { Repeating } \\
\text { unit }\end{array}$ & $\begin{array}{c}\text { Size } \\
\text { fragments }\end{array}$ \\
\hline MN21 & 1567132 & 8 & CCACT & $254-290$ \\
MN23 & 1565219 & 8 & CACC & $219-302$ \\
MS2 & 782947 & 3 & CA12 & $290-312$ \\
MS5 & 30333 & 3 & CAT10 & $173-215$ \\
MS6 & 2835596 & 14 & TGA19 & $194-298$ \\
MS7 & 152239 & 2 & TAA22 & $349-388$ \\
MS8 & 1561308 & 13 & TGTA7 & $284-560$ \\
MSP1-B2 & 1157742 & 7 & Putaporntip & $352-460$ \\
MSP1-B10 & & & et al. (2002) & $226-329$ \\
\hline
\end{tabular}

$a$ : GenBank accession NC_009913.1 (Rezende et al. 2009), chromosome location determined at ncbi.nlm.nih.gov.

of Rondônia (RO) (sample I) and one from the state of Roraima (RR) (sample H). Due to the selective action of CQ on the young trophozoite (ring) stage (Chotivanich et al. 2001), only samples that contained between $50-70 \%$ of the total parasite forms at this stage using short-term in vitro culture were evaluated. The CQ-response of the isolates was measured as released plasmodium lactate dehydrogenase using the DELI test and the $50 \%$ inhibitory concentration $\left(\mathrm{IC}_{50}\right)$ values (Chehuan et al. 2013). Samples with $\mathrm{IC}_{50}$ values greater than $100 \mathrm{nM}$ were considered to be CQ-Rt. This study was approved by the Ethical Committee of Clementino Fraga Filho University Hospital, Federal University of Rio de Janeiro, state of Rio de Janeiro (approval 42746/2012).

Nine neutral markers (Table I), two TRs (MN21 and MN23), five MS (MS2, MS5, MS6, MS7, MS8) and two highly polymorphic blocks of MSP-1 (MSP1-B2 and MSP1-B10) were evaluated by using primers, as described previously (Putaporntip et al. 2002, Feng et al. 2003, Rezende et al. 2009, 2010). These markers have been used previously in studies on the $P$. vivax population structure and diversity in the Brazil endemic area (Rezende et al. 2009, 2010) and in other endemic areas, showing the extent of their polymorphisms (Imwong et al. 2005, Koepfli et al. 2009, 2011).

Plasmodial DNA was extracted from dried blood spots using the QIAamp DNA Micro kit (Qiagen, USA). To increase the quantity of the DNA, parasite DNAs were subjected to whole-genome amplification using a REPLI-g Mini kit (Qiagen) according to the manufacturer's instructions. The markers were amplified using polymerase chain reaction (PCR), according to a previously described methodology (Putaporntip et al. 2002, Feng et al. 2003, Rezende et al. 2009, 2010). The amplified sequences were separated using capillary electrophoresis in an automatic DNA sequencer (MegaBACE, Amersham Biosciences, USA) and the lengths of the products were determined with reference to internal size standards (MegaBACETM ET550-R, Amersham Biosciences) using MegaBACE ${ }^{\text {TM }}$
Fragment Profiler v.1.2 software (Amersham Biosciences). The highest peak in the electropherogram was defined as the predominant allele and additional alleles were considered when the corresponding peak heights were at least $1 / 3$ of the height of the predominant allele in the same sample. In all cases, the minimal peak height for an allele to be considered was set to 100 arbitrary fluorescence units. The predominant allele in each locus per isolate was used to determine gene diversity using the Arlequin 3.0 software that calculated the expected heterozygosity $\left(H_{\mathrm{E}}\right)$, i.e., the probability that a pair of alleles randomly obtained from the population differs from each other.

The six CQ-sensitive (CQ-S) isolates and five CQRt isolates had $\mathrm{IC}_{50}$ medians of $26.35 \mathrm{ng} / \mathrm{mL}$ (7.3-66.4) and $152.8 \mathrm{ng} / \mathrm{mL}$ (108.8-211.8), respectively. The use of multiple molecular markers showed the usefulness and reproducibility of parasite genotyping, as it could be compared with the population studies performed in Brazil using the same panel of markers (Rezende et al. 2009, 2010, Araujo et al. 2012). The $H_{\mathrm{E}}$ average of CQ-Rt samples $\left(H_{\mathrm{E}}=0.528\right)$ and CQ-S samples $\left(H_{\mathrm{E}}=0.594\right)$ were comparable because the difference was not statistically significant (Wilcoxon rank sum test, $\mathrm{p}=0.57$ ). Fortynine alleles were detected among the samples genotyped using nine loci: the more diverse ( $>6$ alleles) were MSP1B10, MS7 and MS5, while the more conserved $(<4$ alleles) were MS2 and MN23 (Table II). Allelic frequencies ranged from $8.3-80 \%$ among the studied samples. For some samples, two alleles were detected at a particular locus: CQ-S F (MSP-1 blocks 2 and 10), CQ-S 85 (MS5),

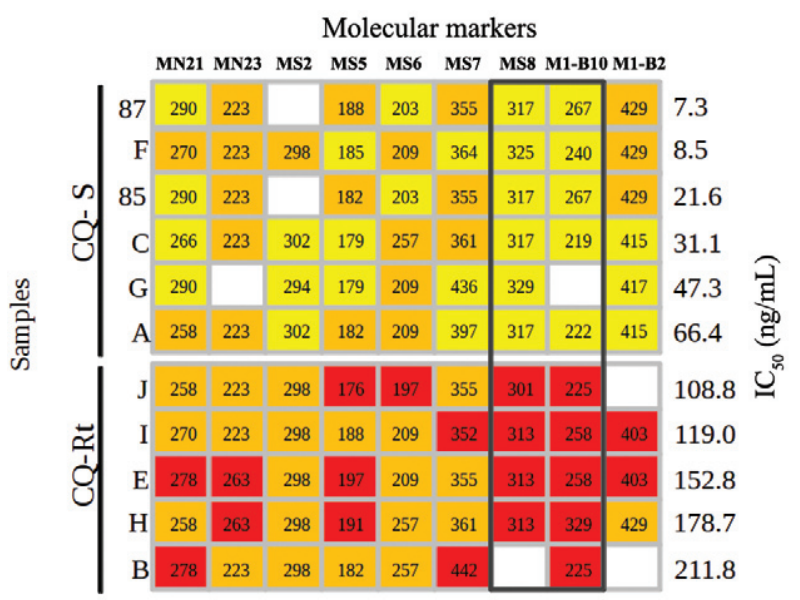

Genetic variability of chloroquine sensitive (CQ-S) and CQ-resistant (CQ-Rt) Plasmodium vivax assessed using tandem repeats (MS21 and MN23), microsatellites (MS2, MS6, MS7 and MS8) and polymorphic antigens, MSP-1 block 2 and 10 (M1-B2 and M1-B10). Predominant alleles (highest peaks on electropherogram) shared by CQ-S and CQ-Rt phenotype are showed in orange, alleles exclusive of CQ-S phenotype in yellow and exclusive of CQ-Rt phenotype in red. Numbers indicate the size of amplified fragment in base pairs. Samples are showed in increasing order of $50 \%$ inhibitory concentration $\left(\mathrm{IC}_{50}\right)$ (right side). Black rectangle highlighted the two markers showing all alleles as phenotype-exclusive. Blank rectangles represent fail in polymerase chain reaction amplification. 
TABLE II

Fragment sizes and allele frequency from Plasmodium vivax isolates from endemic area de Amazon Basin

\begin{tabular}{|c|c|c|c|c|c|c|c|c|c|}
\hline \multirow{2}{*}{$\begin{array}{l}\text { Allele } \\
\text { number }\end{array}$} & \multicolumn{2}{|c|}{$\begin{array}{c}\text { Tandem repeats } \\
\operatorname{Size}^{a}(\%)\end{array}$} & \multicolumn{5}{|c|}{$\begin{array}{l}\text { Microsatellites } \\
\operatorname{Size}^{a}(\%)\end{array}$} & \multicolumn{2}{|c|}{$\begin{array}{l}\text { Surface antigen } \\
\text { Size }^{a}(\%)\end{array}$} \\
\hline & MN21 & MN23 & MS2 & MS5 & MS6 & MS7 & MS8 & MSP1-B2 & MSP1-B10 \\
\hline 1 & $258(27.3)$ & $233(80)$ & 294 (11) & $176(8.3)$ & $197(8.3)$ & $352(8.3)$ & $294(9.1)$ & 403 (20) & $219(8.3)$ \\
\hline 2 & $266(9)$ & $263(20)$ & 298 (67) & 179 (16.7) & 203 (16.7) & $355(33.3)$ & $301(9.1)$ & $415(30)$ & $222(8.3)$ \\
\hline 3 & $270(18.2)$ & - & $302(22)$ & $182(25)$ & 209 (41.7) & $361(16.7)$ & $313(27.3)$ & 417 (10) & 225 (16.7) \\
\hline 4 & $278(18.2)$ & - & - & $185(16.7)$ & $240(8.3)$ & $364(8.3)$ & 317 (36.4) & 429 (40) & $240(8.3)$ \\
\hline 5 & $290(27.3)$ & - & - & $188(16.7)$ & 257 (25) & $397(8.3)$ & $325(9.1)$ & - & $243(8.3)$ \\
\hline 6 & - & - & - & $191(8.3)$ & - & $436(8.3)$ & $329(9.1)$ & - & $252(8.3)$ \\
\hline 7 & - & - & - & $197(8.3)$ & - & $442(8.3)$ & - & - & $258(16.7)$ \\
\hline 8 & - & - & - & - & - & $445(8.3)$ & - & - & $267(16.7)$ \\
\hline 9 & - & - & - & - & - & - & - & - & $329(8.3)$ \\
\hline $\begin{array}{l}\text { Samples } \\
\text { amplified }\end{array}$ & 11 & 10 & 9 & 11 & 11 & 11 & 10 & 9 & 10 \\
\hline$H_{\mathrm{E}} \mathrm{CQ}-\mathrm{S}$ & 0.80 & 0.00 & 0.63 & 0.72 & 0.61 & 0.77 & 0.50 & 0.61 & 0.71 \\
\hline$H_{\mathrm{E}} \mathrm{CQ}-\mathrm{Rt}$ & 0.67 & 0.48 & 0.0 & 0.80 & 0.64 & 0.72 & 0.37 & 0.44 & 0.64 \\
\hline
\end{tabular}

a: fragment size in base pair and its frequency; CQ-Rt: chloroquine resistant; CQ-S: CQ-sensitive; $H_{\mathrm{E}}$ : expected heterozygosity.

CQ-S C (MS7 and MS8), CQ-S A (MS6) and CQ-R H (MSP1-B10) (Table III). Interestingly, despite similar genetic variability between CQ-S and CQ-Rt isolates, multiple alleles per locus were identified in four out six CQ-S isolates, whereas this was only found in one CQ-Rt sample, suggesting a higher variability among CQ-S $P$. vivax samples. Of note, the different alleles of MS8 and MSP1B10 loci were not shared by the two phenotypes (Figure). Particularly for the MS8 locus, allele 317 was detected in 4/6 (67\%) CQ-S phenotype samples and allele 313 in 3/4 (75\%) CQ-Rt phenotype samples: all of which were from different states of the Amazon Basin (RO, AM and RR). This locus is located on chromosome 13 at $166 \mathrm{bp}$ from the 5 ' end of a gene encoding a hypothetical conserved protein with phosphatidylinositol phosphate kinase activ-

TABLE III

Multiple alleles at a particular locus

\begin{tabular}{llll}
\hline $\begin{array}{l}\text { Molecular } \\
\text { marker }\end{array}$ & \multicolumn{2}{c}{ Samples } & Alleles \\
\hline MS5 & CQ-S & 85 & $182 / 185$ \\
MS6 & & A & $209 / 240$ \\
MS7 & & C & $361 / 445$ \\
MS8 & & C & $249 / 317$ \\
MSP1-B2 & F & $415 / 429$ \\
MSP1-B10 & & F & $240 / 243$ \\
MSP1-B10 & CQ-Rt & H & $252 / 329$ \\
\hline
\end{tabular}

CQ-Rt: chloroquine resistant; CQ-S: CQ-sensitive. ity and at 1,942 bp from the 3' end of a gene encoding nuclear transport factor 2 (Rezende et al. 2010). CQ-Rt phenotype exclusive alleles found in this study have also been described in other states of the Amazon Basin, such as Amapá, Pará and Mato Grosso (Rezende et al. 2010), suggesting that the CQ-Rt parasite population could be distributed in other regions of the Amazon Basin.

In conclusion, we found that CQ-S and CQ-Rt $P$. vivax parasites are genetically different. Although the level of genetic diversity was similar in the two phenotypes, both phenotypes could be differentiated by at least two loci. Furthermore, these phenotype-specific alleles were identified in different endemic areas of Brazil, suggesting the potential of resistance spreading across the whole Amazon Basin. Further studies should be performed with a larger number of samples to confirm these findings. Whether these loci are under linkage disequilibrium and involve their flanking genes to impart resistance warrants further investigation.

\section{REFERENCES}

Alecrim MG, Alecrim W, Macedo V 1999. Plasmodium vivax resistance to chloroquine (R2) and mefloquine (R3) in Brazilian Amazon Region. Rev Soc Bras Med Trop 32: 67-68.

Araujo FC, de Rezende AM, Fontes CJ, Carvalho LH, de Brito CFA 2012. Multiple-clone activation of hypnozoites is the leading cause of relapse in Plasmodium vivax infection. PLoS ONE 7: e49871.

Arnott A, Barry AE, Reeder JC 2012. Understanding the population genetics of Plasmodium vivax is essential for malaria control and elimination. Malar J 11: 14.

Chehuan YF, Costa MR, Costa JS, Alecrim MG, Nogueira F, Silveira H, Brasil LW, Melo GC, Monteiro WM, Lacerda MV 2013. In vitro chloroquine resistance for Plasmodium vivax isolates from the western Brazilian Amazon. Malar J 12: 226. 
Chotivanich K, Silamut K, Udomsangpetch R, Stepniewska KA, Pukrittayakamee S, Looareesuwan S, White NJ 2001. Ex-vivo short-term culture and developmental assessment of Plasmodium vivax. Trans $R$ Soc Trop Med Hyg 95: 677-680.

de Brito CFA, Ferreira MU 2011. Molecular markers and genetic diversity of Plasmodium vivax. Mem Inst Oswaldo Cruz 106 (Suppl. I): 12-26.

Duraisingh MT, Cowman AF 2005. Contribution of the $p f m d r l$ gene to antimalarial drug-resistance. Acta Trop 94: 181-190.

Feng X, Carlton JM, Joy DA, Mu J, Furuya T, Suh BB, Wang Y, Barnwell JW, Su XZ 2003. Single-nucleotide polymorphisms and genome diversity in Plasmodium vivax. Proc Natl Acad Sci USA 100: 8502-8507.

Fidock DA, Nomura T, Talley AK, Cooper RA, Dzekunov SM, Ferdig MT, Ursos LM, Sidhu AB, Naudé B, Deitsch KW, Su XZ, Wootton JC, Roepe PD, Wellems TE 2000. Mutations in the P. falciparum digestive vacuole transmembrane protein PfCRT and evidence for their role in chloroquine resistance. Mol Cell 6: 861-871.

Gonçalves LA, Cravo P, Ferreira MU 2014. Emerging Plasmodium vivax resistance to chloroquine in South America: an overview. Mem Inst Oswaldo Cruz 109: 534-539.

Imwong M, Pukrittayakamee S, Grüner AC, Rénia L, Letourneur F, Looareesuwan S, White NJ, Snounou G 2005. Practical PCR genotyping protocols for Plasmodium vivax using Pves and Pvmsp1. Malar J 4: 13.

Kerlin DH, Boyce K, Marfurt J, Simpson JA, Kenangalem E, Cheng Q, Price RN, Gatton ML 2012. An analytical method for assessing stage-specific drug activity in Plasmodium vivax malaria: implications for ex vivo drug susceptibility testing. PLoS Negl Trop Dis 6: e1772.

Koepfli C, Mueller I, Marfurt J, Goroti M, Sie A, Oa O, Genton B, Beck HP, Felger I 2009. Evaluation of Plasmodium vivax genotyping markers for molecular monitoring in clinical trials. $J$ Infect Dis 199: 1074-1080.

Koepfli C, Ross A, Kiniboro B, Smith TA, Zimmerman PA, Siba P, Mueller I, Felger I 2011. Multiplicity and diversity of Plasmodium vivax infections in a highly endemic region in Papua New Guinea. PLoS Negl Trop Dis 5: e1424.
Orjuela-Sánchez P, da Silva NS, da Silva-Nunes M, Ferreira MU 2009. Recurrent parasitemias and population dynamics of Plasmodium vivax polymorphisms in rural Amazonia. Am J Trop Med Hyg 81: 961-968.

Pratt-Riccio LR, Chehuan YF, Siqueira MJ, Alecrim MG, BiancoJúnior C, Druilhe P, Brasseur P, Ferreira-da-Cruz MF, Carvalho LJ, Daniel-Ribeiro CT 2013. Use of a colorimetric (DELI) test for the evaluation of chemoresistance of Plasmodium falciparum and Plasmodium vivax to commonly used anti-plasmodial drugs in the Brazilian Amazon. Malar J 12: 281.

Price RN, Douglas NM, Anstey NM 2009. New developments in Plasmodium vivax malaria: severe disease and the rise of chloroquine resistance. Curr Opin Infect Dis 22: 430-435.

Putaporntip C, Jongwutiwes S, Sakihama N, Ferreira MU, Kho WG, Kaneko A, Kanbara H, Hattori T, Tanabe K 2002. Mosaic organization and heterogeneity in frequency of allelic recombination of the Plasmodium vivax merozoite surface protein-1 locus. Proc Natl Acad Sci USA 99: 16348-16353.

Rezende AM, Tarazona-Santos E, Couto AD, Fontes CJ, de Souza JM, Carvalho LH, Brito CF 2009. Analysis of genetic variability of Plasmodium vivax isolates from different Brazilian Amazon areas using tandem repeats. Am J Trop Med Hyg 80: 729-733.

Rezende AM, Tarazona-Santos E, Fontes CJ, Souza JM, Couto AD, Carvalho LH, Brito CF 2010. Microsatellite loci: determining the genetic variability of Plasmodium vivax. Trop Med Int Health 15: 718-726.

Rieckmann KH, Davis DR, Hutton DC 1989. Plasmodium vivax resistance to chloroquine? Lancet 2: 1183-1184.

Santana Filho FS, Arcanjo AR, Chehuan YM, Costa MR, MartinezEspinosa FE, Vieira JL, Barbosa MD, Alecrim WD, Alecrim MD 2007. Chloroquine resistant Plasmodium vivax, Brazilian Amazon. Emerg Infect Dis 13: 1125-1126.

WHO - World Health Organization 2010. Global report on antimalarial efficacy and drug resistance. Available from: who.int/malaria/ world_malaria_report_2010/worldmalariareport2010.pdf.

WHO - World Health Organization 2013. World Malaria Report 2013. Available from: who.int/malaria/publications/world_malaria report 2013/en/. 